Revue des patrimoines

\title{
La bibliothèque de Mathurin Jousse : une tentative de reconstitution
}

Patrick Le Bœuf

\section{(2) OpenEdition}

Journals

Édition électronique

URL : http://journals.openedition.org/insitu/1111

DOI : 10.4000/insitu. 1111

ISSN : 1630-7305

Éditeur

Ministère de la culture

Référence électronique

Patrick Le Bœuf, «La bibliothèque de Mathurin Jousse : une tentative de reconstitution », In Situ [En ligne], 1 | 2001, mis en ligne le 30 juin 2014, consulté le 30 avril 2019. URL : http:// journals.openedition.org/insitu/1111; DOI : 10.4000/insitu.1111

Ce document a été généré automatiquement le 30 avril 2019

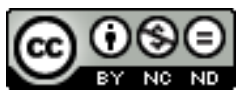

In Situ Revues des patrimoines est mis à disposition selon les termes de la licence Creative Commons Attribution - Pas d'Utilisation Commerciale - Pas de Modification 4.0 International. 


\section{La bibliothèque de Mathurin Jousse : une tentative de reconstitution}

\section{Patrick Le Bœuf}

\section{(fig. $\left.\mathbf{n}^{\circ} \mathbf{1}\right)$}

Figure 1

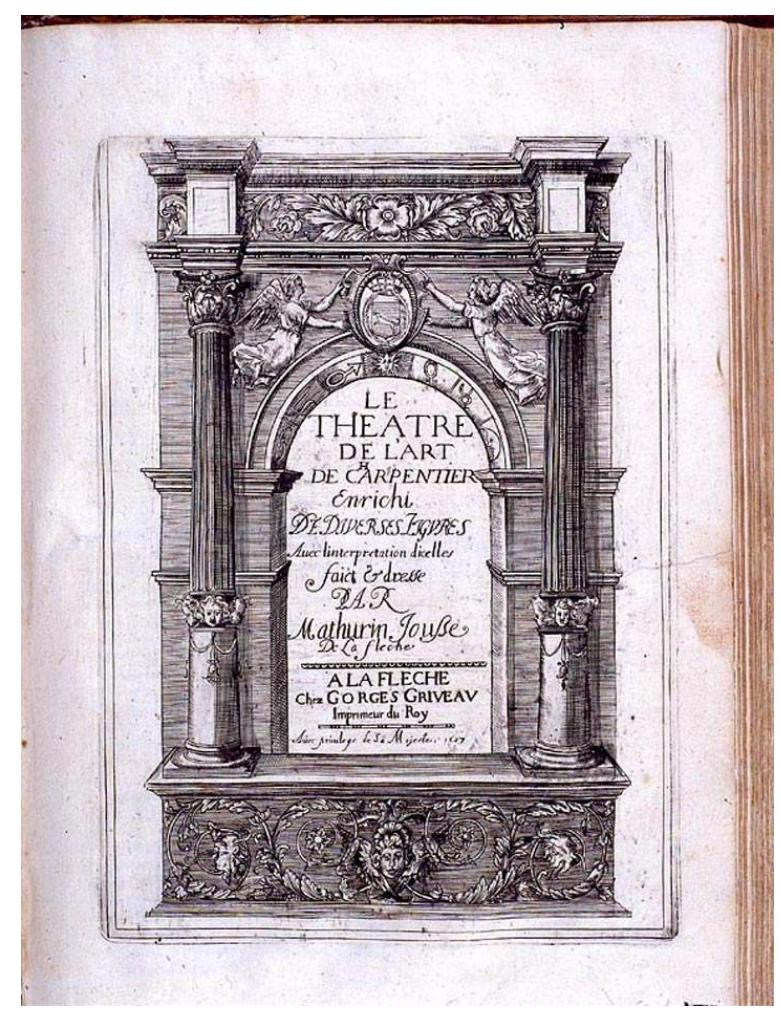

Frontispice du Théâtre de l'art de charpentier, La Flèche, 1627. Phot. Inv. François Lasa @ Inventaire général, ADAGP, 1995 

l'inventaire après décès des biens de Françoise Le Royer, veuve de Mathurin Jousse, en date du 21 décembre 1652. Cet inventaire consacre une part non négligeable (117 items) à la bibliothèque de feu Mathurin Jousse, qu'il est ainsi possible de reconstituer dans ses grandes lignes. Cette bibliothèque apporte un éclairage intéressant sur la personnalité de ce notable du début du XVIIe siècle, qui a laissé plusieurs traités d'architecture et de perspective.

Voici tout d'abord la transcription littérale de la section de l'inventaire consacrée à la bibliothèque de Mathurin Jousse :

Plus dans un autre coffre fermant de clef c'est trouvé les livres suivans.

[1] Premier les Estats et Empires du monde in-fol. veau noir.

[2] La vie des saincts en un thome in-fol. imparfaicte lettre gothicque veau verd.

[3] La conference des ordonnances royaux in-fol. veau verd.

[4] L'hystoire des roys de France commençant par Pharamond et finissant a Charles sept en un thome in-fol. veau rouge.

[5] La Mere des hystoires lettre gothique in-fol. veau noir.

[6] L'astronomicque de Jacques Bazantin Ecossois in-fol. velin.

[7] Le premier et second thome de Pline in $-4^{\circ}$ veau noir.

[8] La vocation des magiciens $4^{\circ}$ velin.

[9] Les six thomes de l'histoire de France par La Serre et Mathieu $4^{\circ}$ velin.

[10] La chymie de Crolius $4^{\circ}$ velin.

[11] Le triomphe de la pieté $4^{\circ}$ velin.

[12] Les ephemerides de Jean Stade latt. $4^{\circ}$ velin.

[13] La troisieme partye de l'honneste femme $4^{\circ}$ velin.

[14] Les vies et opuscules de Plutarche en 4 thomes $4^{\circ}$ velin.

[15] Le ramas de pluzieurs histoires $4^{\circ}$ velin.

[16] La somme des pechés.

[17] La theorye des planettes $4^{\circ}$ vel.

[18] Le chalendryer des bergers $4^{\circ}$ vel.

[19] Le petit atlas iiij $4^{\circ}$ velin.

[20] L'epithome de Pigray $4^{\circ}$ velin.

[21] Cœlum philosophorum per Philipum Ulstadium in- $8^{\circ} \mathrm{rub}$. vell.

[22] Le secretaire françois $8^{\circ}$ vel.

[23] La pirotegnye de Vanocio $4^{\circ}$ vel.

[24] Euclidij [sic)] elementorum per Carolum Malapertium in- $8^{\circ}$ vel.

[25] [Speriatriæ ?)] Laurencii Nucij $4^{\circ}$ mag. vel.

[26] Les essays des merveilles de la nature par François $4^{\circ}$ velin.

[27] La recherche des droitz de la Couronne de France $4^{\circ}$ velin.

[28] Le teatre de l'agriculture $4^{\circ}$ vel. 
[29] L'abregé de l'histoire de France avec les portraicts des roys fol. vel.

[30] Les trois livres en un thome d'Albert avec figures de lettres $4^{\circ}$ vel.

[31] La magie naturelle in- $16^{\circ}$ vel.

[32] Le combat de Geneve $8^{\circ}$ vel.

[33] Les recreations des mathemat. $4^{\circ}$ vel.

[34] Le traicté des anges et demons par Labourie in- $8^{\circ}$ vel.

[35] Les antiquités d'Anjou par Hiret $8^{\circ}$ vel.

[36] L'architecture de Savot $4^{\circ}$ vel.

[37] Les quadrains historiques de la Bible avec les figures $4^{\circ}$ vel.

[38] Le manuel de la Confrairie in- $8^{\circ}$ vel.

[39] La nullité de la religion reformee $4^{\circ}$ vel.

[40] La chyromencie et phisionomye de [un blanc] in- $4^{\circ}$ vel.

[41] Les familieres instructions de la chyromence et phisionomye par Belot curé de Milmontz $4^{\circ}$ vel.

[42] Le livre de Roch Baillif $4^{\circ}$ vel.

[43] Les jugemens astronomicques sur les nativités par Ferrier in- $8^{\circ}$ vel.

[44] Le sommaire de la doctrine chrestienne en schÿmes par [Voissard ?)] $8^{\circ}$ vel.

[45] Le Polidor de Virgille $8^{\circ}$ vel.

[46] L'arithmeticque de Chauvet $4^{\circ}$ vel.

[47] Le Char[illisible]t $8^{\circ}$ veau rouge.

[48] Les amblemes d'Alciat avec figures $4^{\circ}$ vel.

[49] Le Florentin des artz et sciences $4^{\circ}$ vel.

[50] La geometrie de Bulens $4^{\circ}$ mag. vel.

[51] L'architecture de Sagredo $4^{\circ}$ vel.

[52] Le microcosme taille-douce $4^{\circ}$ vel.

[53] Les ephemerides d'Adrian Vlaco latt. $4^{\circ}$ vel.

[54] Le Forcadel sur Euclide $4^{\circ}$ vel.

[55] Le marechal expert $4^{\circ}$ vel.

[56] L'hystoire generalle des plantes et herbes $8^{\circ}$ vel.

[57] Le banquet de Platon $8^{\circ}$ vel.

[58] Le banquet et apres-disner du conte [durreste ?)] $8^{\circ}$ vel.

[59] Le Rabelays reformé $4^{\circ}$ vel.

[60] La geometrie praticque par Boulenger $4^{\circ}$ vel.

[61] La mathematologie de Robert d'Andigné latt. $4^{\circ}$ vel.

[62] L'uzage du quadran par Tarde $4^{\circ}$ vel.

[63] L'epithome de Vitreuve par Gardet $4^{\circ}$ vel.

[64] La cosmographie d'Apian latt. $4^{\circ}$ vel.

[65] Le troisieme livre des orloges de Jean Vello latt. $4^{\circ}$ vel. 
[93] Neuf livres de Du Cerceau dont le 1er contient les bastimens de France et maisons royalles relyé en veau rouge fol. 2. contient les bastimens champestres. 3. contient cinquante bastimens tous differens in-fol. vel. // 4. contient les portes et cheminees fol. vel. 5. contient la perspective $4^{\circ}$ vel. 6 . contient le [Coneil ?)] positive et perspective $4^{\circ}$ vel. 7. contient pour les menuisiers et cherpentiers $4^{\circ}$ vel. 8 . la perspective in- $8^{\circ}$ vel. 9 . contient les bastimens encor $12^{\circ}$ vel.

[66] Le traicté de l'horlogiographie de Zÿpres $8^{\circ}$ vel.

[67] L'arithmeticque et algebre de Stevin $8^{\circ}$ vel.

[68] La geometrie et uzage du compas de proportion par Couette $4^{\circ}$ vel.

[69] L'uzage de l'astrolabe par Jacquinot doré sur tranche $4^{\circ}$ vel.

[70] La geometrie et arithmeticque de Zacquerze [?)] $4^{\circ}$ vel.

[71] La geometrye de Bouelles $4^{\circ}$ vel.

[72] Les discours militaires de [Prissac ?)] $4^{\circ}$ vel.

[73] Les antiquités des villes et chasteaux de France $8^{\circ}$ vel.

[74] Glareani geographia $4^{\circ}$ vel.

[75] Euclides ex Campano fol. vel.

[76] La geometrye de Pelletier $4^{\circ}$ vel.

[77] L'hystoire des possedees de Flandre $4^{\circ}$ vel.

[78] L'uzage du compas de proportion par Henrion $4^{\circ}$ vel.

[79] Les secrez et merveilles de la nature par Veicker $4^{\circ}$ vel.

[80] La doctrine chrestienne sans intitullé $8^{\circ}$ vel.

[81] La bienseance et conversation humaine latt. et françois $8^{\circ}$ vel.

[82] Les epitectes de La Porte $4^{\circ}$ vel.

[83] Alexis Piedmontois $8^{\circ}$ vel.

[84] Les regles de la Congregation N. Dame in-12 ${ }^{\circ}$.

[85] Prutenii motuum cœlestium $4^{\circ}$ velin.

[86] Quatorze livres de Viator latt. françois avec figures par led. deffunct Jousse $4^{\circ}$ vel.

[87] Philbert de L'Horme imparfaict fol. mag. vel.

[88] Clavius super Euclidem $8^{\circ}$ vel.

[89] Les metamorphozes d'Ovide en vers et figures $8^{\circ}$ vel.

[90] Paradoxes ou traictés phisicques des pierres et pierreries $4^{\circ}$ vel.

[91] L'abregé des poids et mezures par De Bedes [?)] in-12 vel.

[92] Plus dix- sept livres traitant d'architecture, de serrurier et cherpentier fol. vel. par led. deffunct Jousse.

[94] Corthoneze de l'architecture des bastimens de Rome ital. fol. vel.

[95] Hieroniæ spiritalium $4^{\circ}$ vel.

[96] La geometrie, spherre, compas et barrois des cours de la comette par Oronse $4^{\circ}$ vel.

[97] Le blazon des armes des plus nobles hommes de France fol. vel.

[98] Les fortiffications de Flament $4^{\circ}$ vel. 
[99] Les mathematicques du meme autheur $4^{\circ}$ vel.

[100] Le recuil de pluzieurs machines militaires par Françoys Thybourel $4^{\circ}$ vel.

[101] Les fortiffications d'Errard fol. vel.

[102] Les fortiffications de Meynier fol. vel.

[103] Les fortiffications de Villechevalyer fol. vel.

[104] Les fortiffications de Vegece fol. vel.

[105] Les fortiffications et architecture artifices de Petr. Cathaneo [un blanc)].

[106] La perspective de Jacques Perret fol. vel.

[107] Albert Duret fol. vel.

[108] La maniere de bien bastir par Le Muet fol. vel.

[109] La regle generalle d'architecture par De Brosses fol. vel.

[110] L'architecture de Cathaneo fol. vel.

[111] Le premier thome de l'architecture de Philbert de L'Horme fol. veau.

[112] L'architecture de Vitreuve fol. vel.

[113] Les œuvres de [pere Chirnoy ?)] fol. veau rouge.

[114] L'astrologie de Ptolomee ital. $4^{\circ}$ veau noir.

[115] Mardelle sur Euclide $4^{\circ}$ vel.

[116] Oronse Forcadel $4^{\circ}$ vel.

[117] Les mathematicques d'Oronse Finisse traictant du compas de proportion $8^{\circ}$ vel.

Si l'on excepte les œuvres de Jousse lui-même ([86], [92]), on dispose d'un corpus de 115 titres qui peuvent se répartir thématiquement de la manière suivante :

\begin{tabular}{|l|l|l|l|}
\hline Agronomie & Auteurs anciens & Géographie politique & Minéralogie \\
Algèbre & Botanique & Géométrie & Ouvrages illustrés \\
Architecture & Chimie & Gnomonique & Peinture \\
Arithmétique & Chiromancie & Histoire & Philosophie \\
Art militaire & Divers et indéterminés & Histoire naturelle & Poliorcétique \\
Artes dictaminis & Droit & Mécanique & Pyrotechnie \\
Astrologie & Ésotérisme & Médecine & Religion \\
Astronomie & Géographie physique & Métrologie & \\
\hline
\end{tabular}

\section{Religion : [2] [11] [16] [44] [80] [84]?}

dont : ouvrages de controverse : [32] [39] [59]

démonolâtrie, possession : [8]-[77] [34]

12 items, soit $10,43 \%$

\section{Philosophie : [57] [58]?}

2 items, soit moins de $1 \%$

Ésotérisme, astrologie etc. : [18] [31]? [43]

dont chiromancie : [40] [41]

5 items, soit $4,35 \%$ 
Chimie : [10] 4 items, soit 3, $48 \%$

Histoire et géographie politique : [1] [4] [5] [9] [14] [15] [29] [35] [97]

9 items, soit $7,83 \%$

Géographie physique : [19] [64] [74]

3 items, soit $2,60 \%$

Droit : [3] [27]

2 items, soit moins de $1 \%$

Métrologie : [91]

1 item, soit moins de $1 \%$

Ouvrages illustrés : [37] [48] [52] [89] [107]?

5 items, soit $4,35 \%$

Histoire naturelle, botanique, minéralogie : [7] [56] [90]

3 items, soit $2,60 \%$

Arithmétique et algèbre : [33] [46] [61] [67] [70] [99]

6 items, soit $5,21 \%$

Géométrie : [24] [50] [54] [70] [75] [88] [96] [106] [115]

dont : arpentage : [60] [71] [76] [116]

usage du compas de proportion : [68] [78] [117]

usage du cadran : [62]

17 items, soit $14,78 \%$

Astronomie : [6] [17] [25]? [69] [85] [114]

dont : éphémérides : [12] [53]

8 items, soit $6,96 \%$

dont : recettes et "trucs" pratiques : [49]? [79]? [83] [100]

5 items, soit $4,35 \%$

Pyrotechnie : [23] [100]

2 items, soit moins de $1 \%$

Médecine : [20] [21] [42]

3 items, soit 2,60\%

Agronomie : [28]

1 item, soit moins de $1 \%$

Mécanique : [95]

1 item, soit moins de $1 \%$

Gnomonique : [50] [65] [66]

3 items, soit 2,60\%

Peinture : [30] [107]? 
2 items, soit moins de $1 \%$

Architecture : [36] [51] [63] [73] [87] [93] [94] [108] [109] [110] [111] [112]

12 items, soit $10,43 \%$

Poliorcétique et art militaire : [72] [98] [100] [101] [102] [103] [104] [105] [106]?

9 items, soit 7,83\%

Divers et indéterminés : [13] [38] [45] [47]? [55] [113]?

6 items, soit $5,21 \%$

Auteurs anciens : [7] [14] [57]

dont : Euclide : [24] [54] [75] [88] [115]

Vitruve : [63] [112]

13 items, soit $11,30 \%$

NB : L'addition de tous ces pourcentages donne un résultat supérieur à $100 \%$ puisqu'un certain nombre de titres ressortissent à plusieurs catégories à la fois.

Au moment où l'inventaire a été établi, il ne semble pas que les livres aient été classés : leur énumération ne suit aucun ordre thématique. On peut toutefois penser que cette situation résulte de déclassements postérieurs au décès de Mathurin Jousse : çà et là en effet, on décèle des " noyaux ", des " nébuleuses " thématiques. L'exemple le plus frappant en est la poliorcétique, condensée sur les items $n^{\circ}$ [98] à [105], ou bien, dans une moindre mesure, les items [60] à [71] qui tournent tous plus ou au moins autour de la géométrie, de l'horométrie et de l'usage des instruments tels que cadran, astrolabe, etc.

Ce qui frappe au premier abord, c'est l'absence quasi totale de titres littéraires, même parmi les auteurs de l'Antiquité (Ovide étant présent, semble-t-il, plus en raison de la qualité iconographique de l'exemplaire possédé par Jousse que pour le texte même). On remarque ensuite l'inexistence de traités de serrurerie, alors que la serrurerie était l'activité première de Jousse et qu'il a rédigé un ouvrage sur cette matière. À noter, en revanche, l'abondance de traités d'architecture et de poliorcétique (qui peuvent d'ailleurs être regroupés dans une seule catégorie) ainsi que de géométrie : c'étaient visiblement les domaines qui préoccupaient le plus Mathurin Jousse. Il s'intéressait également à l'astronomie et à la gnomonique, et se montrait soucieux d'écrire dans un style élégant (artes dictaminis). Les événements du passé et la situation politique du monde de son temps retenaient aussi son attention.

La personnalité plus intime de Jousse transparaît elle aussi à travers sa bibliothèque, et nous réserve quelques surprises : il semble avoir éprouvé une certaine attirance pour l'ésotérisme, l'astrologie, la chiromancie - attirance qui n'allait toutefois pas jusqu'à une passion approfondie. Il accordait un grand intérêt aux questions religieuses, et notamment à la controverse, à la réfutation du protestantisme. Mais le plus étonnant, c'est la présence de cet étrange libelle dont les deux tomes n'étaient matériellement plus rangés ensemble lorsque le notaire a dressé son inventaire, et qui relate un cas de possession et de sorcellerie survenu en Flandre en 1623 ([8]-[77]). Pourquoi s'intéresser à une province aussi éloignée ? Dans ce contexte, on peut s'étonner de ne trouver aucun écho de l'affaire de Loudun de 1634, plus proche géographiquement et qui avait suscité la parution d'un très grand nombre d'opuscules, notamment chez Georges Griveau, l'éditeur fléchois attitré de Mathurin Jousse. 
Les lieux d'édition ne nous apprennent pas grand-chose. Il semble surprenant de ne pas relever plus d'éditions purement locales : en dehors des œuvres de Jousse lui-même, toutes publiées à La Flèche chez Georges Griveau, on ne trouve guère que deux éditions fléchoises : l'ouvrage de controverse d'Honorat Nicquet imprimé par Louis Hébert, et celui d'un " gentilhomme breton " anonyme, imprimé par Jacques Rezé, et une édition angevine : Des antiquités d'Anjou de Jean Hiret, paru chez A. Hernault. Mais peut-être les titres non identifiés ressortissent-ils partiellement à la production éditoriale locale ? Mentionnons en outre quelques ouvrages provenant de petits éditeurs locaux géographiquement très éloignés du pays de Mathurin Jousse : les deux traités de Flamant ([98] et [99]), tous deux imprimés à Montbéliard, l'ouvrage de Thybourel ([100]), édité à Pont-à-Mousson, et celui du père Leurechon ([33]), peut-être édité dans la même ville.

6 L'étude des dates d'édition se révèle en revanche plus fructueuse. Sur les trente-neuf éditions dont l'identification est quasi certaine (soit le tiers de la bibliothèque de Jousse), on en dénombre 18 à coup sûr antérieures à 1620 (dont 9 datant très probablement du XVIe siècle, parmi 14 dont on peut seulement dire qu'elles sont antérieures à 1615), 15 qui s'échelonnent entre 1620 et 1630 , et 5 qui sont postérieures à 1630 . Compte tenu du fait qu'il reste tout de même beaucoup d'éditions non identifiées, et considérant qu'un ouvrage édité à une date donnée peut très bien n'avoir été acheté que bien plus tard, il parait assez raisonnable d'affirmer, avec toute la prudence qui s'impose, que Mathurin Jousse a constitué l'essentiel de sa bibliothèque entre 1620 et 1630, soit une période assez courte et qui correspond, ce qui n'est nullement surprenant, à sa pleine maturité.

7 Voilà pour les quelques conclusions matérielles que l'on peut tirer de cet inventaire. Mais une investigation certainement plus riche consisterait à l'exploiter par rapport à l'œuvre de Jousse lui-même, pour étudier la manière sont il s'est servi de ses lectures, notamment dans le domaine de l'architecture et de la poliorcétique, pour élaborer ses propres théories. Une étude plus précise de la personnalité des auteurs de ces ouvrages pourrait également éclairer, par ricochet, celle de Jousse : combien d'entre eux étaient jésuites, ou proches des jésuites ? Qu'est-ce qui a déterminé le choix de tel titre plutôt que tel autre au moment de l'achat?

Il convient par ailleurs de souligner que, vu le laps de temps qui s'est écoulé entre le décès de Mathurin Jousse (1645) et l'établissement de l'inventaire (1652), il n'est pas totalement impensable qu'un certain nombre d'ouvrages effectivement possédés de son vivant par le maître serrurier fléchois ne figurent pas dans cette liste, considération que tend à corroborer l'impression de " déclassement " post mortem subi par ce fonds d'ouvrages. Cette remarque de prudence ne retire rien à l'intérêt de ce document, même si elle engage à appréhender avec une certaine circonspection l'éclairage qu'il jette sur ce qu'a pu être, dans la France provinciale du début du XVIIe siècle, la culture générale et technique d'un " honnête homme " avant la lettre.

\section{Annexes}

(1) Il peut s'agir de plusieurs ouvrages portant des titres voisins, mais on remarque dans [BNF] : Les Estats, empires et principautés du monde, représentés par la description des pays, mœurs des habitans, richesse des provinces, les forces, le gouvernement... par le sr D.T.V.V. - Paris, P. Chevalier, 1619. - In-4 .

\section{(2) (3) (4) Ouvrages non identifiables}

(5) Il s'agit bien évidemment de La mer des histoires, " best-seller " dès sa première édition à Paris en 1488 et constamment réédité durant toute la première moitié du XVIe siècle. 
Selon Brunet, il s'agit de " la traduction des Rudimenta novitiorum [...] faite par un chanoine de Mello en Beauvaisis, qui a continué cette chronique jusqu'au règne de Louis $\mathrm{XI}$, mais qui ne s'est pas nommé. " Le grand nombre de rééditions interdit une identification plus précise de l'exemplaire que possédait Mathurin Jousse.

(6) Bassantin, Jacques. - Astronomia J. Bassantini, ... - Coloniæ Allobrogum : apud J. Tornæsium, 1599. - In-fol. [BNF] ; ou : Astronomicque discours, par Jacques Bassantin, ... À Lion : par J. de Tournes, 1557. - In-fol. (2e éd. : Genève, 1631) [BNF]. Le notaire prenant grand soin de préciser la langue des ouvrages lorsqu'ils ne sont pas rédigés en français, il est probable qu'il s'agit de la traduction française.

(7) Exemplaire non identifiable.

(8) et (77) Ces deux items, cités à grande distance l'un de l'autre par le notaire, représentent en réalité 2 tomes d'un seul et même ouvrage : Histoire veritable et memorable de ce qui s'est passé sous l'exorcisme de trois filles possedees ès païs de Flandres, en la descouverte et confession de Marie de Sains... et Simone Dourlet... et autres... Extraict des memoires de messire Nicolas de Momorenci comte d'Estarre,... Sebastien Michaelis,... RPF François Donsieux,... mis en lumiere par I. Le Normant sieur de Clairemont,... - Paris : O. de Varennes, 1623. - 2 vol. in- ${ }^{\circ}$ [BNF]. Le tome I s'intitule : Les confessions de Didyme, sorcière pénitente, avec les choses qu'elle a déposées touchant la synagogue de Sathan l'Antéchrist... ; le tome II : De la vocation des magiciens et magiciennes par le ministere des demons...

196 (9) Ouvrage non identifié. Pierre Matthieu a écrit une histoire de France souvent rééditée, mais en 7 livres et non 6 et sans la collaboration d'un autre auteur dénommé La Serre (qui ne semble pas devoir être identifié avec Puget de La Serre).

(10) Oswald Croll est l'auteur d'un traité de chimie intitulé Basilica chymica très fréquemment réédité, soit dans son texte latin, soit en traduction française, qui est plus probablement la version que possédait Jousse, pour la même raison que ci-dessus : La royale chymie de Crollius traduitte en françois par J. Marcel de Boulenc. - Lyon : P. Drouet, 1624. - In- $8^{\circ}$; ibid., 1627. - In- $8^{\circ}$; Paris : M. Henault, 1633. - In- $8^{\circ}$; Rouen : J. Berthelin, 1634. - In- $8^{\circ} ;$ Rouen : J. Osmont, 1634. - In- $8^{\circ}[\mathrm{BNF}]$.

(12) Les Éphémérides de Jean Stade ont été fréquemment rééditées dans la seconde moitié du XVIe siècle : Coloniæ Agrippinæ : apud hæredes A. Birckmanni, 1556. - In- $4^{\circ}$. ; ibid., 1650. - In- $4^{\circ}$; ibid., 1581. - In-4 $4^{\circ}$; Lugduni : apud S. Berand et S. Michaelem, 1585. In $-4^{\circ} \ldots$ [BNF].

(13) Du Bosc, Jacques. - L'honneste femme. 3e partie. - Paris : Pierre Billaine, 1632. - In-8 [BL] ; Paris : A. Courbé, 1636. - In-4 $4^{\circ}$ [BNF]. La 1re et la 2e parties ont été plusieurs fois rééditées entre 1633 et 1636 à Paris, soit chez J. Jost soit chez A. Soubron. Le fait que le notaire spécifie qu'il s'agit d'un in-4 $4^{\circ}$ semble indiquer la réédition de 1636 plutôt que la 1ère édition de 1632, mais cette précision est à considérer avec beaucoup de précaution.

(14) Exemplaire non identifié.

(15) Ouvrage non identifiable.

(16) Un trop grand nombre d'ouvrages de la Renaissance portent ce titre pour que celui-ci soit identifiable.

(17) On pourrait en dire autant de cet item-ci, mais le fait que le notaire prenne toujours la peine d'indiquer les auteurs des livres qu'il recense lorsque leurs noms figurent sur la page de titre et que les ouvrages dont il ne donne que le titre ont effectivement été 
publiés sans mention d'auteur, incite à donner la préférence au traité de James Hume : La theorie des planettes, contenant l'usage et construction de toutes sortes de tables astronomiques... Avec la response aux premières invectives du sieur Morin,... - Paris : J. Dugast, 1637. In- $8^{\circ}$ [BNF, CNAM].

(18) Exemplaire non identifiable, le Calendrier des bergers étant un autre " best-seller " de la Renaissance.

(19) Ouvrage non identifiable.

(20) Pigray, Pierre. - Epitome des préceptes de médecine et chirurgie, avec ample déclaration des remèdes propres aux maladies, par P. Pigray. - Parisiis : M. Orry, 1609. - In-8 ; 3e éd. Lyon : impr. de S. Rigaud, 1661. - In- $8^{\circ}$ [BNF]. Est également paru en latin : Parisiis : apud viduam M. Orry, $1612 .-$ In- $8^{\circ}$ [BNF].

(21) Ulstadt, Philipp. - Coelum philosophorum, seu de secretis naturce liber, Philippo Ulstadio,... authore - Argentorati : arte et impensa J. Grienynger, 1528. - In-fol. [BNF]; Argentorati : excud. Iacobus Cammerlander, 1535 [Cambridge]. Cœelum philosophorum, seu secreta naturce, id est quomodo non solum e vino, sed etiam ex omnibus metallis, fructibus, carne, ovis, radicibus, herbis et aliis quam plurimis, quinta essentia sive aqua vitee ad conservationem humani corporis debeat educi, liber tum medicis ac chirurgis tum pharmacopolis, imo et... ex variis authoribus... Parisiis : apud V. Gautherot, 1544. - In- $8^{\circ}$ [BNF]. Coelum philosophorum seu liber de secretis naturce, per Philippum Ulstadium ex variis autoribus accurate selectus... - Lugduni : apud G. Rouillum, 1553. - In-16 ${ }^{\circ}$ [BNF, Cambridge] ; ibid., 1557 [Cambridge] ; ibid., 1572 [BNF, Cambridge]. En français : Le ciel des philosophes. - Paris : on les vend par Vivant Gaultherot, 1550 [Cambridge].

(22) Ouvrage non identifiable, un trop grand nombre de livres portant ce titre.

(23) Biringuccio, Vanoccio. - La pyrotechnie, ou art du feu, contenant dix livres, ausquels est amplement traicté de toutes sortes et diversité de minières, fusions et séparations des métaux... composée par le seigneur Vanoccio Biringuccio,... et traduit d'italien en françois par feu maistre Jacques Vincent. — Paris : C. Fremy, 1556. - In-4º ; Paris : G. Jullien, 1572. - In- $4^{\circ} ;$ Rouen : J. Cailloüe, 1627. - In- $4^{\circ}$ [BNF, CNAM].

211 (24) Malapert, le père Charles. - Euclidis elementorum libri sex priores quorum demonstrationes... accomodavit Carolus Malapertius,... - Duaci : typis M. Belleri, 1620. In- $16^{\circ}$ [BNF].

212 (25) Ouvrage non identifié.

213 (26) François, René (pseudonyme d'Étienne Binet). - Essay des merveilles de nature et des plus nobles artifices, pièce trés-nécessaire à tous ceux qui font profession d'éloquence, par René François, prédicateur du roy. - Rouen : R. de Beauvais, 1621. In- $4^{\circ}$ [BNF]. Nombreuses rééditions entre 1622 et 1632 à Rouen chez J. Osmont, ainsi qu'à Paris chez J. Dugast.

(27) Ouvrage non identifié.

(28) Beaucoup d'ouvrages portent ce titre. Peut-être s'agit-il de celui d'Olivier de Serres : Théâtre d'agriculture et mesnage des champs. - Paris, 1600. - In-fol. [CNAM] ?

(29) Ouvrage non identifiable.

(30) Il ne peut guère s'agir que du De pictura de Leone Battista Alberti, seul ouvrage de cet auteur à se présenter en 3 livres ; mais il ne semble pas qu'il ait été traduit en français du vivant de Mathurin Jousse. De pictura præstantissima et numquam satis laudata arte libri 
tres absolutissimi Leonis Baptistæ de Albertis,... Basileæ, 1540. - In-8 [BNF]. La pittura di Leon Battista Alberti, tradotta per M. Lodovico Domenichi. - Vinegia : G. Giolito de Ferrari, 1547. - In- $8^{\circ}[\mathrm{BNF}]$.

intitulé " Simple et véritable discours de ce qui s'est passé en la conférence commencée à Caen entre quelques ministres et le P. Gontier, jésuite " faict par D. L., gentil-homme breton. - Paris [i. e. La Flèche] : J. Rezé, 1606. - In-8 [BNF]. Jacques Rezé a été, de 1604 jusqu'à sa mort en 1618, l'imprimeur officiel du Collège des Jésuites de La Flèche (selon Émile Pasquier et Victor Dauphin, Imprimeurs et libraires de l'Anjou. — Angers, 1932, p. 338 sqq.).

(40) Chiromantia. 1 : Physiognomia ex aspectu membrorum hominis. 2 : Periaxiomata de faciebus signorum... - Coloniæ : apud P. Regnault, 1543. - In- $8^{\circ}$ [BNF]. Vraye... chyromancie et phisionomie par le regard des membres de l'homme [par J. ab Indagine, trad. par A. Du Moulin]. - Paris : I. Villery, [1620 ?]. - In-8 ${ }^{\circ}$ [BL].

(41) Belot, Jean, curé de Milmonts. - Familières instructions pour apprendre les sciences de chiromance et physionomie... avec un traité des divinations, augures et songes par $\mathrm{M}$. Jean Belot,... Paris : N. Bourdin, 1624. - In- $8^{\circ}$ [BNF]. 
(42) Roch Le Baillif de La Rivière a composé plusieurs traités de médecine, édités soit à Rennes (Pierre Le Bret, M. Logeroys) soit à Paris (A. L'Angelier, G. Aliot) entre 1577 et 1623. Il est impossible de déterminer duquel il est ici question.

(43) .Ferrier, Auger ou Oger. - Jugemens astronomiques sur les nativitez, avec l'epitome

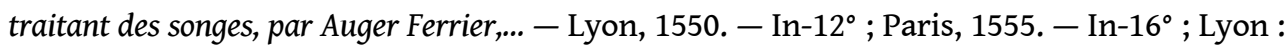
J. de Tournes, 1582. - In- $16^{\circ}$; Rouen : N. Lescuyer, 1583. - In-16 ${ }^{\circ}$ [BNF et Bibliographie générale de l'astronomie jusqu'en $1880 \mathrm{de} \mathrm{J.-C.} \mathrm{Houzeau} \mathrm{et} \mathrm{A.} \mathrm{Lancaster].}$

(44) Ouvrage non identifié.

(45) Il s'agit d'un des traités de Polydorus Vergilius, mais duquel ? peut-être du De inventoribus rerum?

(46) Chauvet, Jacques. - Méthodiques institutions de la vraye et parfaicte arithmétique de Jacques Chauvet. Souvent réédité : à Paris (C. Roger, 1585, in-16² ; L. Bruneau, 1606, in- $8^{\circ}$; H. Hunot, 1606 , in- $8^{\circ}$; M. Daniel, 1619 , in- $8^{\circ}$ ) ou à Rouen (A. Ouyn, 1631, in- $8^{\circ}$; M. de La Motte, 1636 et 1640 , in- $\left.8^{\circ}\right)$. Table générale d'arithmétique composée par Jacques Chauvet,... Paris : H. de Marnef et Vve G. Cavellat, 1581. - In-fol. [BNF].

(47) Ouvrage non identifié.

(48) Trop souvent réédité pour que l'exemplaire soit identifiable.

(49) Ouvrage non identifié.

(50) Bullant, Jean. - Petit traicté de geometrie et d'horologiographie pratique, par Jehan Bullant,... - À Paris : chez G. Cavellat, 1562. - In- $4^{\circ}$ [BNF] ; ibid., 1564. - In-4 ${ }^{\circ}$ [BL, CNAM]. Geometrie et horlogiographie pratique, contenant la description, fabrication et usage des horloges solaires, par Jehan Bullant,... augmentée de la propriété et usage des quadrans de l'invention d'Oronce Finé,... et de Pierre Appian,... Le tout exposé facilement par Claude de Boissière,... - Paris : D. Cavellat, 1608. - In-4 [BNF].

(51) Sagredo, Diego de. - Raison d'architecture antique, extraicte de Vitruve et aultres anciens architecteurs, nouvellement traduit d'espaignol en françoys à l'utilité de ceulx qui se delectent en édifices. - Paris : Simon de Colines, [entre 1526 et 1537]. - In- $4^{\circ}$; ibid., 1542. - In- $4^{\circ}$; Paris : G. Gourbin, 1555. - In $4^{\circ}$ [BNF]. De l'architecture antique demonstree par raisons tres faciles, pour l'utilité de ceux qui se délectent en édifices... Traduit d'espagnol en françois... Paris : D. Cavellat, 1608. - In-4 $4^{\circ}$ [BNF].

(52) Gravure non identifiable.

(53) Vlacq, Adriaan. - Ephemerides motuum cœlestium ad annos vulgaris æræ 1633, 1634, 1635 et 1636 summa diligentia in luminarium motibus et zyzygiis ex tabulis lansbergianis ab Adriano Vlacco,... in reliquis planetis ex tabulis rudolphinis a Joanne Keplero,... supputatæ, cum instructione super earum usu. - Goudæ : excudebat P. Rammusenius, 1632. - In- $4^{\circ}$ [BNF].

(54) Forcadel, Pierre. Trad. - [Les éléments]. - [S. 1. n. d.]. Les six premiers livres des Éléments... - Paris : C. Perier, 1566. Les septième, huictième et neufième livres des Éléments... - Paris : C. Perier, 1565 [BNF].

(55) Ouvrage non identifié.

(56) Ouvrage non identifié.

(57) Exemplaire non identifiable.

(58) Ouvrage non identifié. 
(59) Garasse, François. - Le Rabelais réformé par les ministres et nommément par Pierre Du Moulin, ministre de Charanton, pour response aux bouffonneries insérées en son livre de la vocation des pasteurs. - Doué : impr. de F. Ruffin, $1620 .-$ In- $8^{\circ} ;$ Brusselle : impr. de C. Girard, 1619, 1620, 1621. - In-8 ${ }^{\circ}$ [BNF]. La suite du Rabelais réformé sur les impertinentes réponses de $\mathrm{M}$. Pejus aux demandes de $\mathrm{M}$. Durand, avec la coupelle mystique des ministres au sieur de La Pierre, déministré. - Au Palais, jouxte la copie imprimée à Mer, 1624. - In-8 ${ }^{\circ}$ [BNF].

(60) Boulenger, Jean. - La géométrie pratique du sieur Boulenger,... ou nouvelle méthode de toiser et arpenter toutes sortes de grandeurs... - Paris : G. Charpentier, 1634. - In- $4^{\circ}$ [BNF]. La géométrie pratique des lignes, des superficies et des corps... mise en lumière par le sieur Boulenger,... - Paris : l'auteur, 1630. - In- $4^{\circ}$; Paris : S. Cramoisy, 1640. - In- $4^{\circ}$ [BNF].

(61) Andigné, Robert d'. - Compendium sive technologia in consuetudines Britanniæ, a Roberto Dandinaco,... compositum nuperque in lucem emissum. - Redonis : apud G. Cleray, 1545 [BNF]

(62) Tarde, Jean. - Les usages du quadrant à l'esguille aymantée, divisé en deux livres : le premier donne la cognoissance du quadrant; le second, les usages, utilitez et services qui en peuvent estre tirez... par Jean Tarde,... - Paris : J. Gesselin, 1621, 1623, 1638. - In-4 ${ }^{\circ}$ [BNF].

(63) Gardet, Jan. - Epitomé ou extrait abrégé des 10 livres d'architecture de Marc Vitruve Pollion,... par Jan Gardet,... et Dominique Bertin, aveq les annotations sur les plus difficiles passages de l'auteur... - Tolose : par G. Boudeville, 1559. - In- $4^{\circ}$; Paris : G. Buon, 1567, 1568. $-\mathrm{In}-4^{\circ}[\mathrm{BNF}]$.

(64) Benewitz, Peter, dit Petrus Apianus. Sa Cosmographia sive descriptio universi orbis a été trop fréquemment rééditée tout au long du XVIe siècle pour que l'exemplaire soit identifiable.

(65) Voellus, le père Jean. - De horologiis sciothericis libri tres, Joanne Voello,... auctore, ... [Scholia autoris in eosdem tres de horologiis libros non minoris æstimanda, quam libri ipsi]. - Turnonii : apud C. Michaelem et T. Soubron, 1608. - In-4 [BNF].

(66) Ouvrage non identifié, ainsi que l'auteur allégué. S'agirait-il d'une erreur de lecture du notaire pour un ouvrage en langue flamande dont le titre contiendrait le mot cijpher, transcrit Zÿpres de manière erronée?

(67) Stevin, Simon. - L'arithmetique de Simon Stevin,... Aussi l'algebre... - Leyde : impr. de C. Plantin, 1585. -2 tomes en 1 vol. in- $8^{\circ}$. L'arithmetique de Simon Stevin,... revue, corrigée et augmentée de plusieurs traictez et annotations par Albert Girard, samielois... - Leyde : impr. des Elzeviers, 1625. - In- $8^{\circ}$. Les œuvres mathematiques, de Simon Stevin, ... I : L'arithmetique, contenant : les computations des nombres arithmetiques ou vulgaires ; aussi l'algebre, avec les equations des cinq quantitez... II : La cosmographie... [etc.]. - Leyde : impr. de B. et A. Elsevier, 1634. - 6 tomes en 1 vol. in-fol. [BNF]

(68) Ouvrage non identifié.

(69) L'usage de l'astrolabe, avec un petit traicté de la sphère, de Dominique Jacquinot, a fréquemment été réédité à Paris : J. Gazeau, 1545. - In- $4^{\circ} ;$ G. Cavellat, 1558, 1559. - In-8 ${ }^{\circ}$ ; impr. de H. de Marnef et G. Cavellat, 1576. - In- $8^{\circ}$; Vve Cavellat, 1598. - In-8 $8^{\circ}$; H. de Marnef, 1617. - In- $8^{\circ}$ [BNF]. Il est le plus souvent associé à : Une amplification de l'usage de l'astrolabe, de Jacques Bassantin. 

[BNF].

(70) Ouvrage non identifié. On trouve toutefois, d'un certain Zacarie, " professeur ès mathématiques " : Traicté d'arithmétique, géométrie, avec l'art d'arpanter et mesurer toutes superficie [sic] de terre... Nouvellement mis en lumière par le sieur Zacarie,... - À Paris : chez N. Rousset, 1618. - In- $8^{\circ}$. Livre d'arithmétique et geometrie, avec l'art d'arpenter et mesurer toutes superficies de terre. Nouvellement mis en lumière par le sieur Zacarie,... Revu, corrigé et augmenté de nouveau. Ensemble un traicté de la boussole... - À Paris : chez N. Rousset, 1625. - In- $8^{\circ}$ [BNF]. Le nom de l'auteur est difficilement lisible sur l'inventaire ; pourrait-il s'agir d'une déformation de " Zacarie " ?

(71) Bouelles, Charles de. - Livre singulier et utile, touchant l'art praticque de geometrie, composé nouvellement en françoys, par maistre Charles de Bouvelles,... - Paris : imprimé par S. de Colines, 1542. - In- $4^{\circ}$. Geometrie practique, composée par... Charles de Bouelles, et nouvellement par luy reveue, augmentée et grandement enrichie. - Paris : impr. de Regnaud Chaudière et Claude son fils, 1547. - In- $4^{\circ}$; Paris : ibid., 1551. - In- $4^{\circ}$; Paris : G. Cavellat, 1555. - In- $4^{\circ}$; Paris : H. de Marnef et G. Cavellat, 1566. - In- $4^{\circ}$. Geometrie practique composée par... Charles de Boüelles,... avec un traicté des mesures geometriques, des hauteurs accessibles ou inaccessibles et de toutes choses pleines ou profondes... par M. Jean-Pierre de Mesmes... Plus l'art de mesurer toutes superficies rectilignes, tiré des Elemens d'Euclide, par M. Jean Des Merliers d'Amiens... - Paris : D. Cavellat, 1605. - In- $8^{\circ}$; ibid., 1608. - In- $8^{\circ}$ [BNF].

(72) Ouvrage non identifié. L'auteur appartient-il à la famille de Cossé-Brissac ?

(73) Ouvrage non identifiable.

(74) Loriti, Heinrich, dit Glareanus. - Henrici Glareani,... de geographia liber unus, ab ipso authore jam novissime recognitus. - Apud Friburgum Brisgaudiæ : excudebat J. Faber, 1530. - In- $4^{\circ}$; Venetiis : apud J. A. de Nicolinis de Sabio, 1538, 1539. - In- $4^{\circ}$; Parisiis : apud G. Rikart, 1542. - In $-4^{\circ}$; Parisiis : apud H. de Marnef et G. Cavellat, 1577. - In $-4^{\circ}$ [BNF].

(75) Campano, Giovanni. Trad. Comment. - Elementa geometrice Euclidis, latine, cum annotationibus Campani. - Venetiis, 1482. - In-fol. Euclidis Megarensis... opera a Campano interprete fidissime tralata... - Venetiis : [s. n.], 1509. - In-fol. Geometricorum elementorum libri XV. Campani,... in eosdem commentariorum libri XV... [Jacobus Faber edidit]. - Parisiis : in officina H. Stephani, [1516]. - In-fol. Euclidis,... elementorum geometricorum lib. XV, cum expositione Theonis in priores XIII..., Campani in omnes et Hypsidis,... in duos postremos... Basileæ : J. Hervagius, 1537. - In-fol. ; Basileæ : J. Hervagius et B. Brand, 1558. - In-fol.

(76) Peletier, Jacques, dit Peletier du Mans. - Jacobi Peletarii,... de usu geometrice liber unus... - Parisiis : apud E. Gorbinum, 1572. - In-4 ${ }^{\circ}$. De l'usage de geometrie, par Jacques Peletier, ...- Paris : G. Gourbin, 1573. - In- $4^{\circ}[\mathrm{BNF}]$.

(78) Henrion, Didier. - L'usage du compas de proportion, par D. Henrion,... Nouvellement revû, corrigé et augmenté d'une seconde partie... - Rouen : D. Du Chesne, 1564. - In-8 $8^{\circ}$; 2e éd. Paris : M. Mondière, $1624 .-$ In- $8^{\circ} ; 4$ e éd. - Paris : S. Thiboust, $1631 .-$ In- $8^{\circ} ; 5 e$ éd. Rouen : J. Boulley, 1637. - In- $8^{\circ}$ [BNF].

(79) Wecker, Johann Jacob. - Les secrets et merveilles de nature, recueillis de diversautheurs et divisez en XVII livres, par Jean Jacques Vuecker,... [trad. par Gabriel Chappuis et autres]. - À Lyon : par B. Honorati, 1586. - In-8 $8^{\circ}$ À Rouen : chez C. Le Villain, 1633. - In-8 ${ }^{\circ}$ [BNF] ; Rouen : chez C. Malassis, 1639. - In- $8^{\circ}$ [Giovanna Grassi, Union catalogue of printed books of 15 th, 16th \& 17th centuries in European astronomical observatories]. De secretis libri XVII. Ex 
variis authoribus collecti, methodiceque digesti \& aucti. - Basileæ : sumptibus J. Regis ; excud. J. R. Genath, 1662. - In-8 $8^{\circ}$ [Giovanna Grassi, op. cit.]. fol. [93.2] : Livre d'architecture de Jacques Androuet Du Cerceau, auquel sont contenues diverses ordonnances de plants et elevations de bastiments pour seigneurs, gentilshommes et autres qui voudront bastir aux champs ; mesmes en aucuns d'iceux sont desseignez les bassez courts... aussi les jardinages et vergiers... - Paris : impr. pour J. Androuet Du Cerceau, 1582. - In-fol. ; ibid., 1615. - In-fol. [93.3] : Livre d'architecture de Jacques Androuet Du Cerceau, contenant les plans et dessaings de cinquante bastimens tous differens, pour instruire ceux qui désirent bastir... - Paris : [s. n.], 1559. - In-fol. [93.4] : Second livre d'architecture par Jacques Androuet Du Cerceau, contenant plusieurs et diverses ordonnances de cheminees, lucarnes, portes, fonteines, puis et pavillons... avec les desseings de dix sepultures toutes differentes. - Paris : impr. pour J. Androuet Du Cerceau, 1561. - In-fol. ; Paris : impr. de A. Wechel, 1561. — In-fol. [93.5] : ? 
[93.6] : Leçons de persepctive positive. — Paris : M. Patisson, 1576. — In-fol. [93.7] : ? [93.8] : ? [93.9]:?

(94) Ouvrage non identifié. L'auteur est très probablement Pietro Berrettini, dit Pietro da Cortona.

(95) [95] : Il s'agit des Pneumatica d'Héron d'Alexandrie. Heronis Alexandrini spiritalium liber, a Federico Commandino,... ex grceco nuper in latinum conversus. [Edidit Valerius Spaciolus]. Urbini : [s. n.], 1575. - In- $4^{\circ}$; [Parisiis] : apud A. Gorbinum, 1583. - In- $4^{\circ}$ [BNF, CNAM].

(96) (117) Curieusement, bien qu'Oronce Finé soit un auteur très célèbre et très étudié, ces titres ne semblent se référer à aucune de ses œuvres connues (mais voir [116]). [96] pourrait éventuellement désigner sa Protomathesis.

(97) Ouvrage non identifiable.

(98) Flamand, Claude. - La guide des fortifications et conduitte millitaire pour bien se fortifier et deffendre, de M. Claude Flamand,... - Seconde edition. - Montbéliard : J. Foillet, [s. d.]. In-8 $8^{\circ}[\mathrm{BNF}]$.

(99) Flamand, Claude. - Les mathematiques et geometrie departies en cinq livres... de M. Claude Flamand,... - Seconde edition. - Montbéliard : J. Foillet, [s. d.]. - In- $8^{\circ}$ [BNF].

(100) Thybourel, François. - Recueil de plusieurs machines militaires et feux artificiels pour la guerre et récréation, avec l'alphabet de Trittemius par laquelle chacun... peut promptement composer congruement en latin, aussi le moyen d'escrire la nuict a son amy absent, de la diligence de Franc. Thybourel,... et de Jean Appier, dit Hanzelet,... - Pont-à-Mousson : C. Marchant, imprimeur, 1620. $-\mathrm{In}-4^{\circ}$ [BNF].

(101) Errard, Jean. - La fortification demonstree et reduicte en art, par feu J. Errard,... reveue, corrigee et augmentee par A. Errard, son neveu,... suivant les memoires de l'autheur, contre les grandes erreurs de l'impression contrefaicte en Allemagne,... - Paris : [s. n.], 1620. - In-fol. ; Paris : [s. n.], 1622. - In-fol. [BNF].

(102) Meynier, Honorat de. - Les nouvelles inventions de fortifier les places contre la puissance d'assaillir par traverses, galleries, mines, canons et autres machines de guerre... par Honorat de Meynier,... Le tout representé par figures gravees en taille douce par Crispin de Pas le jeune. Paris : N. Rousset et J. Jacquin, 1626. — In-fol. [BNF].

(103) Ouvrage non identifié.

(104) Ouvrage trop fréquemment réédité pour que l'exemplaire soit identifiable.

(105) Le notaire a confondu Girolamo Cataneo, auteur de l'item [105], avec Pietro Cataneo, auteur de l'item [110]. Le Dell'arte militare de Girolamo Cataneo est paru en italien à Brescia, chez T. Bozzola, en 1571 (in- $4^{\circ}$ ), et chez P. M. Marchetti, en 1584 et 1608 (in- $4^{\circ}$ ) ; dans une traduction latine due à Jean de Tournes (De arte bellica, sive de designandis ac construendis arcibus et propugnaculis), à Genève, chez Jean de Tournes, en 1600 (in- $\left.4^{\circ}\right)$; mais également dans une traduction française, qui est plus probablement celle dont il s'agit ici (cf. supra) : Le capitaine de Jérosme Cataneo, contenant la maniere de fortifier places, assaillir et defendre... Le tout reveu, corrigé et augmenté en plusieurs lieux par l'auteur, et depuis mis en françois [par Jean de Tournes]. - Lyon : J. de Tournes, 1574. - In-4 [BNF]

(106) Perret, Jacques. - Des fortifications et artifices, architecture et perspective de Jacques Perret. [Planches gravées par Thomas de Leu]. - [S. 1.] : [s. n.], [s. d.]. - In-fol. [BNF]. Les fortifications et artifices. Mis en lumière par la veuve et les deux fils de Theodore de Bry. Francfort : Richter, 1602. - In-fol. [CNAM]. L'exemplaire possédé par Jousse constituerait- 
il un état éditorial antérieur, où la perspective était abordée indépendamment de la poliorcétique, état éditorial aujourd'hui absent de [BNF] comme de [CNAM] ?

(107) Paléographiquement, il est impossible de lire autre chose que " Duret " (ou, à l'extrême rigueur, " Durel ", le notaire ayant tendance à donner la même morphologie à ses $t$ et à ses 1 en finale), mais on peut supposer qu'il s'agit en réalité du traité de dessin d'Albrecht Dürer, souvent réédité au cours du XVIe siècle.

(108) Le Muet, Pierre. - Manière de bastir, pour toutes sortes de personnes, par Pierre Le Muet,... - [Paris, 1623]. - In-fol. ; 2de éd. - Paris : F. Langlois, 1647. - In-fol. ; Paris : C. Jombert, [s. d.]. - In-fol. [BNF].

293 (109) Bullant, Jean. - Reigle generalle d'architecture des cinq manieres de colonnes... à l'exemple de l'antique, suivant les reigles et doctrine de Vitruve [par Jean Bullant], reveue et corrigée par M. de Brosse,... - 2e et derniere edition. - Paris : H. de Marnef, 1619. - In-fol. [BNF]. L'erreur d'attribution du notaire vient de ce que le nom de l'auteur ne figure pas sur la page de titre, qui ne fait état que du réviseur, Salomon de Brosse.

294 (110) Cataneo, Pietro (cf. [105]). - I quattro primi libri di architettura, di Pietro Cataneo,... Vinegia : Aldo, 1554. - In-fol. [BNF]. L'architettura di Pietro Cataneo,... alla quale, oltre all'essere stati dall'istesso autore rivisti, meglio ordinati e di diversi disegni e discorsi arricchiti $i$ primi quatro libri per l'adietro stampati, sono aggiunti di più il quinto, sesto, settimo e ottavo libro... - Venetia : Aldo, 1567. - In-fol. [BNF].

(111) Ouvrage non identifiable.

(112) Ouvrage trop souvent réédité pour que l'exemplaire soit identifiable.

(113) Ouvrage non identifié.

(114) Ouvrage trop souvent réédité pour que l'exemplaire soit identifiable.

(115) Le Mardelé, Pierre. - Les quinze livres des éléments geometriques d'Euclide,... traduicts de grec en françois... par P. Le Mardelé,... - Seconde edition. - Paris : D. Moreau, 1632. In- $8^{\circ}[\mathrm{BNF}]$. Les quinze livres des éléments geometriques d'Euclide,... traduits du grec en françois... par Pierre Mardelé,... - Lyon : S. Rigaud, 1645. - In-8 ${ }^{\circ}$ [BNF, CNAM].

(116) Finé, Oronce. - La practique de la geometrie d'Oronce,... traduicte par Pierre Forcadel,... Paris : G. Gourbin, $1570 .-\mathrm{In}-4^{\circ}$; ibid., 1586. - In- $4^{\circ}$ [BNF].

301 (117) cf. [96].

\section{INDEX}

Keywords : on line, electronic journal, ejournal, heritage, history of art, France, architecture, Jousse Mathurin, locksmithing

Mots-clés : en ligne, journal, revue électronique, revue numérique, périodique, patrimoine, histoire de l'art, France, architecture, Jousse Mathurin, serrurerie 


\section{AUTEUR}

\section{PATRICK LE BEUF}

Archiviste-paléographe, Conservateur à la Bibliothèque nationale de France PATRICK.LEBOEUF@bnf.fr 